\title{
Ischemic stroke in a patient with cholangiocarcinoma: a case study
}

\author{
Acidente vascular isquêmico em paciente com colangiocarcionoma \\ Kamon Chaiyasit ${ }^{1}$, Viroj Wiwanitkit ${ }^{2}$ \\ 'Maefahluang University Hospital, Bangkok, Thailand; \\ ${ }^{2}$ Visiting University Professor, Hainan Medical University, Haikou, China. \\ Correspondence: Kamon Chaiyasit; Maefahluang University Hospital, Khlong Tan Nuea; Bangkok 10110 - Thailand; E-mail: kamolchaiyasit@hotmail.com \\ Received 16 May 2012; Received in final form 24 May 2012; Accepted 31 May 2012
}

Dear Editors,

The concurrent neurological disorder among the cancerous patient is an important but forgotten issue. According to a retrospective study by Li et al. ${ }^{1}$ on 10,693 patients, 15 patients experiencing 16 ischemic strokes within the first month after the latest. Interesting, although the stroke is existed in cancerous patient, it is usually not considered in cancer therapy. If stroke occurs, a more complex scenario can be expected and this can be a new problem in holistic management of the cancerous patient. Here, the authors would like to share an experience on a male cholangiocarcinoma patient (stage 4) with the first diagnosis for two years. This patient was undergone chemo-immunotherapy on one first round. On the last week of the cycle, the case complained for the sudden dizziness and weakness. At first, the symptomatic treatment was given but his symptom did not improve. The computerized tomography (CT scan) was further done and showed the suspected infarction or brain metastasis. However, the confirmation by CT scan revealed many areas of ischemic infarction on his brain. This case was finally diagnosed to have an ischemic stroke as a concurrent abnormality. The patient was referred to the neurologist for stroke management. From history review, this case had underlying hypertension and dyslipedemia without a continuous care. As noted, the existence of concurrent ischemic stroke in cancerous patient is not common and can be interesting case report. For some cancers such as head and neck cancer, it is confirmed for increased risk of ischemic stroke $e^{2}$. Based on the literature, this is the first concurrent appearance of cholangiocarcinoma and ischemic stroke. Of interest, cholagiocarcinoma is usually advanced and the patient usually died early, hence, it might be the reason for no previous report. However, this case got the good intensive cancer therapy so the unexpected concurrent neurological disorder can be seen.

\section{References}

1. Li SH, Chen WH, Tang Y, et al. Incidence of ischemic stroke postchemotherapy: a retrospective review of 10,963 patients. Clin Neurol Neurosurg 2006;108:150-156.
2. Lee CC, Su YC, Ho HC, et al. Increased risk of ischemic stroke in young nasopharyngeal carcinoma patients. Int $J$ Radiat Oncol Biol Phys 2011;81:833-838. 